Revue pluridisciplinaire d'études médiévales

\title{
Grivoiserie, pornographie, scatologie : éléments bibliographiques
}

\section{Marie Dupuy et Mathilde Grodet}

\section{(2) OpenEdition}

1 Journals

Édition électronique

URL : http://journals.openedition.org/questes/2682

DOI : 10.4000/questes.2682

ISSN : 2109-9472

Éditeur

Les Amis de Questes

\section{Édition imprimée}

Date de publication : 15 juin 2011

Pagination : 128-134

ISSN : 2102-7188

\section{Référence électronique}

Marie Dupuy et Mathilde Grodet, « Grivoiserie, pornographie, scatologie : éléments bibliographiques » Questes [En ligne], 21 | 2011, document 9, mis en ligne le 15 janvier 2014, consulté le 15 septembre 2020. URL : http://journals.openedition.org/questes/2682 


\section{Éléments bibliographiques}

\section{Dictionnaires et usuels}

BeLmont, Nicole, entrée «Escrementi », in Ruggiero Romano (dir.), Enciclopedia Einaudi, Turin, Einaudi, 1977-1984, t. V.

Dictionary of the Middle Ages, Joseph R. StRAYER (éd.), New York, Charles Scribener's sons, 1982-1989, 13 vol.

Dictionnaire encyclopédique du Moyen Âge, André VAuchez (éd.), Paris, Le Cerf, 1997, 2 vol.

Enzyklopädie des Märchens, Rolf Wihelm BREDNICH (dir.), Berlin/New York, Walter de Gruyter, 1975-[en cours], t. IV, entrée « Exkremente », t. XI, entrée « Puppe : Die beißende P. », t. XII, entrée «Skatologie ».

Grand Larousse Universel, Paris, Larousse, 1991, 15 vol., entrée « scatologie ».

GuIRAud, Pierre, Dictionnaire érotique, Paris, Payot, 1993 (3 édition).

Lexicon des Mittelalters, Munich/Zurich, Artemis/Lexma, 1977-1999, 10 vol.

Rossiaud, Jacques, entrée « sexualité », in Jacques LE GoFf et Jean-Claude SCHMITT (dir.), Dictionnaire raisonné de l’Occident médiéval, Paris, Fayard, 1999.

\section{II. Études historiques}

Amour, mariage et transgressions au Moyen Âge. Actes du colloque des 24, 25, 26 et 27 mars 1983, Amiens, Danielle Buschinger et André Crepin (dir.), Centre d'études médiévales, Göppingen, A. Kümmerle, 1984.

Bologne, Jean-Claude, Histoire de la pudeur, Paris, Hachette, « Pluriel », 1987.

Casagrande, Carla, Vecchio, Silvana, Histoire des péchés capitaux au Moyen Âge, Paris, Aubier, « Collection Historique », 2002 (1 ${ }^{\text {ère }}$ éd. italienne 2000).

FeIXAs, Jean, Pipi, caca, popo : histoire anecdotique de la scatologie, Genève, Liber, 1996.

FLANDRIN, Jean-Louis, Le Sexe et l'Occident. Évolution des attitudes et les comportements, Paris, Seuil, 1981.

FLANDRIN, Jean-Louis, Un Temps pour embrasser. Aux origines de la morale sexuelle occidentale (VI ${ }^{e}-X I^{e}$ siècles), Paris, Seuil, 1983.

GonTHIER, Nicole, «Sanglant Coupaul! » «Orde Ribaude! » Les injures au Moyen Âge, Rennes, Presses Universitaires de Rennes, 2007.

GuerRAnD, Roger-Henri, Les Lieux. Histoire des commodités, Paris, La Découverte, 1986. 
The Invention of Pornography. Obscenity and the Origins of Modernity : 1500-1800, Lynn Avery HunT (dir.), New York, Zone Books, 1993.

JACQUART, Danielle, ThOMAsset, Claude, Sexualité et savoir médical au Moyen Âge, Paris, PUF, « Les chemins de l'Histoire », 8, 1985.

LAPORTE, Dominique G., Histoire de la merde, Paris, Christian Bourgois Éditeur, 1978.

LAqueur, Thomas, La Fabrique du sexe, Essai sur le corps et le genre en Occident,

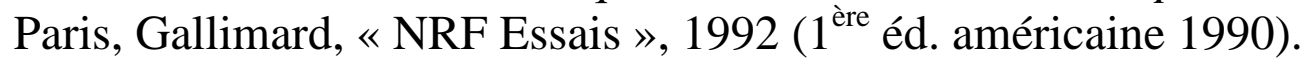

LE Goff, Jacques, L'Imaginaire médiéval, in Un autre Moyen Âge, Paris, Gallimard, 1999, «Quarto », p. 421-770.

Le Goff, Jacques, Truong, Nicolas, Une Histoire du corps au Moyen Âge, Paris, Liana Levi, 2003.

MARTIN, Laurent, "Jalons pour une histoire culturelle de la pornographie en occident », Le Temps des médias, 2003/1 n¹, p. 10-30.

MONESTIER, Martin, Histoire et bizarreries sociales des excréments des origines à nos jours, Paris, Le Cherche Midi, 1997.

PoLY, Jean-Pierre, Le Chemin des amours barbares. Genèse médiévale de la sexualité européenne, Paris, Perrin, 2003.

RiBemont, Bernard, Sexe et amour au Moyen Âge, Paris, Klincksieck, «50 Questions », 2007.

Verdon, Jean, L’Amour au Moyen Âge. La chair, le sexe et le sentiment, Paris, Perrin, 2006.

VoisEnet, Jacques, « Mariage et interdits sexuels au Moyen Âge ( $\mathrm{V}^{\mathrm{e}}-\mathrm{XII}{ }^{\mathrm{e}}$ siècle) », in Sex, Love and Marriage in Medieval Literature and Reality, Thematische Beiträge im Rahmen des $31^{\text {th }}$ International Congress on Medieval Studies an der Western Michigan University (Kalamazoo-USA) 8-12 mai 1996, Greifswald, Reineke, « Wodan », 69, 1996, p. 53-72.

\section{III. Études anthropologique, sociologiques, psychanalytiques}

Agamben, Giorgio, Stanze : parole et fantasme dans la culture occidentale, Paris, Payot et Rivages, « Bibliothèque Rivages », 1994 ( $1^{\text {ère }}$ éd. italienne 1977).

BAKHTINE, Mikhaïl, L'Euvre de François Rabelais et la culture populaire au Moyen Âge et sous la Renaissance, Paris, Gallimard, 1990 (1 $1^{\text {ère }}$ éd. Gallimard 1970/1 ${ }^{\text {ère }}$ éd. russe 1965).

BOURKE, John Gregory, Les Rites scatologiques, Dominique G. LAPORTE (éd.), Paris, PUF, 1981 (1 ${ }^{\text {ère }}$ éd. américaine 1891).

CAstelli, Patrizia, « Il doppio significato. L'ostensione della vulva nel medioevo », 
in S. Bertelli et M. Centanni (dir.), Il gesto nel rito e nel ceremoniale dal mondo antico ad oggi, Florence, Ponte alle Grazia, 1995, p. 199-223.

Douglas, Mary, De la souillure. Essai sur les notions de pollution et de tabou, Paris, Maspero, 1971 ( $1^{\text {ère }}$ éd. anglaise 1966).

Duerr, Hans Peter, Nudité et Pudeur : le mythe du processus de civilisation, Paris, Édition de la Maison des sciences de l'homme, 1998 ( $1^{\text {ère }}$ éd. allemande 1988).

DufRESNE, Jean-Luc, «L'Obscène médiéval », in L'Obscène, Sylvain Floc’H (dir.), Pau, Université de Pau et des pays de l'Adour, "Cahiers de l'Université », 4, 1983, p. 83-94.

ELIAS, Norbert, La Civilisation des mours, Paris, Calmann-Lévy, 1973.

ElliotT, Dyan, Fallen Bodies : Pollution, Sexuality, and Demonology in the Middle Ages, Philadelphia, University of Pennsylvania Press, 1999.

FLEMING, Margaret, "Analysis of a Four-Letter Word», Maledicta: The International Journal of Verbal Aggression, 1 (1977), p. 173-184.

FouCAult, Michel, Histoire de la sexualité, 1, La volonté de savoir, Paris, Gallimard, 1976.

FREUD, Sigmund, « Caractère et érotisme anal » et « Des transpositions pulsionnelles, en particulier dans l'érotisme anal ", in Euvres Complètes, André Bourguignon, Pierre CoteT et Jean LAPLANCHE (éd.), Paris, PUF, 2007 (1 ${ }^{\text {ere }}$ éd. allemande 1908), t. VIII, p. 187-194 et t. XV, p. 53-62.

GAIGNEBET, Claude, Le Folklore obscène des enfants, Paris, Maisonneuve et Larose, 1974.

GoODY, Jack, La Raison graphique : la domestication de la pensée sauvage, Paris, Éditions de Minuit, 1978 ( $1^{\text {ère }}$ éd. anglaise 1977).

GuIRAUD, Pierre, Les Gros mots, Paris, PUF, « Que sais-je? », n¹579, 1975.

INGLIS, David, A Sociological History of Excretory Experience. Defecatory Manners and Toiletry Technologies, Edwin Mellen, Lampeter, « Mellen Studies in Sociology », 30, 2001.

Kristeva, Julia, Pouvoirs de l'horreur : essai sur l'abjection, Paris, Seuil, « Points », 1980.

MAIER, Corinne, L'Obscène. La mort à l'œuvre, La Versanne, Encre Marine, 2004.

PaUlinI, Christian Franz, Heylsame Dreck-Apothecke: wie nemlich mit Koth und Urin die meisten Krankheiten und Schäden glücklich geheilet worden, Stuttgart, Verlag des Herausgebers, 1847 (1 ${ }^{\text {ère }}$ éd. allemande 1696).

RouAYrenc, Catherine, Les Gros Mots, Paris, PUF, « Que sais-je ? », n¹597, 1996.

Vigarello, Georges, Le Propre et le sale. L'Hygiène du corps depuis le Moyen Âge, Paris, Seuil, « Points », 1985. 


\section{Histoire de l'art}

Bartholeyns, Gil, DitTmar, Pierre-Olivier, Jolivet, Vincent, « Des raisons de détruire une image », Images Re-vues, 2 (2006), http://imagesrevues.revues.org/248.

Bartholeyns, Gil, DitTMAR, Pierre-Olivier, Jolivet, Vincent, Image et transgression au Moyen Âge, Paris, PUF, « Lignes d'art », 2008.

BrunA, Denis, Saints et diables au chapeau. Bijoux oubliés du Moyen Âge, Paris, Seuil, 2007.

CAMille, Michael, Images dans les marges. Aux limites de l'art médiéval, Paris, Gallimard, «Le Temps des images », 1997 (1 ${ }^{\text {ère }}$ éd. américaine 1992).

Camille, Michael, The Medieval Art of Love: Objects and Subjects of Desire, Londres, Laurence King, 1998.

Colin-Goguel, Florence, L'Image de l'amour charnel au Moyen Âge, Paris, Seuil, 2008.

GaigneBet, Claude, Lajoux, Jean-Dominique, Art profane et religion populaire au Moyen Âge, Paris, PUF, 1985.

Les Images honteuses, Murielle GagneBin et Julien MiLly (dir.), Seyssel, Champ Vallon, 2006.

MANDABACH, Marisa, "Holy Shit: Bosch's Bluebird and the Junction of the Scatological and the Eschatological in Late Medieval Art », Marginalia, octobre 2010, http://www.marginalia.co.uk/journal/10apocalypse/.

Millet Catherine, "Plutôt le fumier que le trésor; Moderne Art's Excrement Adventure », Art Press, 242 (janvier 1999), p. 27-35.

MüLlER, Markus, "Fonctions du profane et du "ridiculum" dans l'enluminure médiévale ", Histoire de l'art, 29/30 (mai 1995), p. 23-31.

Obscène, obscénités, Steven Bernas et Jamil DaKhLIA (dir.), Paris, L’Harmattan, 2008.

Obscenity : Social Control and Artistic Creation in the European Middle Ages, Jan M. ZIOLKOWSKI (dir.), Leiden, New-York, Köln, Brill, 1998.

Schmitt, Jean-Claude, "L'univers des marges ", in Jacques Dalarun (dir.), Le Moyen Âge en lumière. Manuscrits enluminés des bibliothèques de France, Paris, Fayard, 2002, p. 329-361.

ShaPIRO, Harvey Alan, «Eros in love : Pederasty and Pornography in Greece », in Amy Richlin (dir.), Pornography and Representation in Greece and Rome, NewYork/Oxford/Toronto, Oxford University Press, 1992, p. 53-72.

WIRTH, Jean, Les Marges à drôleries des manuscrits gothiques (1250-1350), Genève, Droz, 2008. 


\section{V. Études littéraires}

Allen, Valerie, On Farting: Language and Laughter in the Middle Ages, Basingstoke, Palgrave Macmillan, 2007.

AubAilly, Jean-Claude, « Le fabliau et les sources inconscientes du rire médiéval », Cahiers de Civilisation Médiévale, XXX (1987), p. 105-117.

BEC, Pierre, Burlesque et obscénité chez les troubadours : le contre-texte au Moyen Âge, Paris, Stock, « Moyen Âge », 1984.

BEuTIN, Wofgang, Sexualität und Obszönität. Eine Literatur psychologische Studie über epische Dichtungen des Mittelalters und der Renaissance, Königshausen \& Neumann, Würzburg, 1990.

Bloch, R. Howard, The Scandal of the Fabliaux, Chicago/Londres, University of Chicago Press, 1986.

Borghi Cedrini, Luciana, La Cosmologia del villano. Secondo testi extravaganti del Duecento francese, Alessandria, Edizioni dell'Orso, 1989.

Bouche, Thérèse, «L'obscène et le sacré ou l'utilisation paradoxale du rire dans le Roman de la Rose de Jean de Meun ", in Thérèse Bouche et Hélène CHARPENTIER (dir.), Le Rire au Moyen Âge dans la littérature et dans les arts, Bordeaux, Presses Universitaires de Bordeaux, 1990, p. 83-95.

BRAET, Herman, "Audigier, de la dissonance comme moyen de dérision », in Jean DufOURnet (dir.), "Si a parlé par moult ruiste vertu». Mélanges de littérature médiévale offerts à Jean Subrenat, Paris, Champion, "Colloques, congrès et conférences sur le Moyen Âge », 1, 2000.

Conformité et déviances au Moyen Âge. Actes du deuxième colloque international de Montpellier, Marcel FAURE (dir.), Montpellier, Centre de recherche interdisciplinaire sur la société et l'imaginaire au Moyen Âge, « Les cahiers du CRISIMA », 2, 1995.

Conlon, Denis Joseph, "La Chanson d'Audigier - A scatological parody of the Chansons de geste edited from Ms. Bibliothèque Nationale, f. fr. 19152 », Nottingham Medieval Studies, XXXIII (1989), p. 21-55.

L'Érotisme au Moyen Âge. Études présentées au troisième colloque de l'Institut d'études médiévales, Bruno Roy (dir.), Montréal, L’Aurore, 1977.

FAIVRE Bernard, "Les rythmes de la farce ou les gestes et les mots », in Jean-Pierre BORDIER (dir.), L'Économie du dialogue dans l'ancien théâtre européen. Actes du colloque du C.E.S.R. (Tours, 26-27 octobre 1995), Paris, Champion, « Le Savoir de Mantice », 4, 1999, p. 33-39.

Fecal Matters in Early Modern Literature and Art. Studies in Scatology, Jeff PERSELS et Russell Ganim (dir.), Aldershot, Ashgate, 2004.

Ferroul, Yves, «Réalités sexuelles et fiction romanesque », in Danielle 
Buschinger (dir.), Les « Realia » dans la littérature de fiction au Moyen Âge. Actes du colloque du Centre d'études médiévales de l'Université de Picardie-Jules Verne, Amiens, Presses du Centre d’Études Médiévales Université de Picardie, « Médiévales », 9, 2000, p. 40-49.

FrapPIER-Mazur, Lucienne, «Marginal Canons : Rewriting the Erotic », in Joan E. DeJean et Nancy K. Miller (dir.), The Politics of Tradition : Placing Women in French Literature, New Haven, Yale University Press, "Yale French Studies », 75, 1988, p. 112-128.

GIER, Albert, " Skatologische Komik in der französischen Literatur des Mittelalters », in Werner SCHRÖDER (dir.), Wolfram-Studien, 7 (1982), p. 154-183.

GRAVDAL, Kathryn, «Vilain et courtois ». Transgressive Parody in French Literature of the Twelfth and Thirteenth Centuries, Lincoln et London, University of Nebraska Press, 1989.

Griffin, Miranda, "Dirty stories: Abjection in the Fabliaux », New Medieval Literatures, 3 (1999), p. 229-260.

HICKS, Eric, «Fabliau et sous-littérature: remarques sur Le Prestre teint », Reinardus, 1 (1988), p. 79-85.

Hicks, Eric, "Le Signe et l'interdit», in Jean-Claude Aubailly et alii (dir.), «Et c'est la fin pour quoy nous sommes ensemble »: hommage à Jean Dufournet. Littérature, histoire et langue du Moyen Âge, Paris, Champion, 1993, t. 2, p. 739-746.

Huston, Nancy, Mosaïque de la pornographie, Paris, Payot, 2004.

KARRAS, Ruth Mazo, Sexuality in Medieval Europe. Doing unto others, New York/Londres, Routledge, 2005.

KoOpmans, Jelle, VerhuycK, Paul, «Les mots et la chose ou la métaphore comme spectacle. Nouvelle étude sur la représentation scénique de l'acte sexuel dans les farces », Versants, 38 (2000), p. 31-51.

LeJeune, Rita, « Hagiographie et grivoiserie. À propos d'un Dit de Gautier le Leu », Romance Philology, 12 (1958-1959), p. 355-365.

Leupin, Alexandre, « Le Sexe dans la langue : la dévoration. Sur Du Con, fabliau du XIII ${ }^{\mathrm{e}}$ siècle de Gautier le Leu », Poétique, 45 (février 1981), p. 91-110.

Mcdonald, Nicola F., Medieval Obscenities, York, York Medieval Press/Boydell Press, 2006.

MelA, Charles, «Un paradoxe littéraire: le Lai du lecheor », Colloquium Helveticum, 5 (1987), p. 59-71.

MenARD, Philippe, Le Rire et le sourire dans le roman courtois en France au Moyen Âge (1150-1250), Genève, Droz, 1969.

Morrison, Susan Signe, Excrement in the Late Middle Ages: Sacred filth and 
Chaucer's fecopoetics, New York, Palgrave Macmillan, 2008.

NAYAR, Sheila J., "Coprus [sic] Christi : the Scatological Tales of the Fabliaux », in Holly A. Crocker (dir.), Comic Provocations : Exposing the Corpus of Old French Fabliaux, New York, Palgrave Macmillan, 2006, p. 63-81.

Neldi, René, Écrivains anticonformistes du Moyen Âge occitan, 1, La Femme et l'amour, Paris, Phébus, 1977.

Le $\mathrm{Nu}$ et le vêtu au Moyen Âge (XII ${ }^{e}$-XIII ${ }^{e}$ siècles). Actes du $25^{\mathrm{e}}$ colloque du CUER MA, 2-3-4 mars 2000, Senefiance $n^{\circ} 47$, Aix-en-Provence, Publications de l’Université de Provence, 2001, p. 382-394.

SCHEIDEGGER, Jean, « Gros mots, gros rires ? Le comique sémiologique de quelques fabliaux scabreux ", in Thérèse Bouche et Hélène CHARPENTIER (dir.), Le Rire au Moyen Âge dans la littérature et dans les arts, Bordeaux, Presses Universitaires de Bordeaux, 1990, p. 309-322.

TISsIER, André, «Évocation et représentation scénique de l'acte sexuel dans l'ancienne farce française », in Maria CHIABO, Federico Doglio et Marina MAYMONE (dir.), Atti del IV colloquio della Société Internationale pour l'Étude du Théâtre Médiéval, Viterbo 10-15 Luglio 1983, Viterbo, Centro studi sul teatro medioevale e rinascimentale, 1984, p. 521-547.

ZiolKowski, Jan, Alain de Lille's Grammar of Sex : The Meaning of the Grammar to a Twelfth-Century Intellectual, Cambridge Mass., The Medieval Academy of America, 1985. 